\title{
Treatment of Huntington Disease
}

\author{
Aleksandar Videnovic, MD, MSc \\ Department of Neurology Parkinson's Disease and Movement Disorders Center Northwestern \\ University Feinberg School of Medicine 710 North Lake Shore Drive, Suite 1106 Chicago, IL \\ 60611 Phone: 312-503-1819 Fax: 312-908-5073 a-videnovic@northwestern.edu
}

\section{Keywords}

Huntington disease; Treatment; Chorea; Antidopaminergic agents; Antipsychotics; Haloperidol; NMDA receptor antagonists; Cognitive dysfunction; Behavioral disturbances; Psychosis; Deep brain stimulation

\section{Introduction}

Huntington disease (HD) is an autosomal dominant, progressive neurodegenerative illness affecting motor function, cognition and behavior. HD is caused by an unstable expanded cysteineadenosine-guanine (CAG) trinucleotide repeat in the IT15 (huntingtin) gene located at the tip of the fourth chromosome (4p16.3) [1]. Age of onset varies from early childhood to late eighties. Peak age of onset is in the forties and fifties. The rate of progression correlates with age of onset and number of CAG repeats, but varies greatly, even within families [2]. Average duration of the illness from onset is 15-17 years.

The clinical triad of HD includes chorea, psychiatric/behavioral disturbances and cognitive decline. Initial HD symptoms of HD vary widely. Motor signs are present in about $60 \%$ of new cases, behavioral problems in $15 \%$, and combination of these two in $25 \%$ cases [3]. The motor disorder of HD is severely disabling and combines involuntary movements, abnormal control of ocular movements and derangements of volitional movements. Chorea is the most common involuntary movement in patients with HD. Chorea tends to diminish with disease progression, as parkinsonism and dystonia emerge $[4,5]$. Irritability and aggressive behavior are frequently present for many years before the onset of motor symptoms. Other common behavioral problems in HD include personality changes, agitation, impulsiveness, anxiety, mania, apathy, social withdrawal and sexual disorders. Symptoms of executive dysfunction are early signs of cognitive decline in HD, and are related to the abnormalities in the frontostriatal networks [6]. Visuospatial processing is affected in patients with HD, and deteriorates more rapidly than memory [7]. HD is diagnosed based on the typical clinical manifestations in conjunction with positive family history, and confirmatory genetic testing.

Significant research efforts are focused on the development of therapies that might modify the course of HD. Although many chemical compounds have shown promise in animal models of HD, no agent has been shown to modify the disease. Therapeutic interventions in HD focus on symptomatic treatment of motor, behavioral and psychiatric disturbances. Tetrabenazine is the only FDA-approved pharmacological agent for treatment of chorea associated with HD. Tetrabenazine binds to the vesicular monoamine transporter (VMAT2), depleting monoamines and serotonin from presynaptic central nervous system neurons.

Disclosure No potential conflicts of interest relevant to this article were reported. 
Chorea usually improves with antidopaminergic medications such as dopamine antagonists or depleters. There is some support in the literature for treatment of chorea with typical and atypical neuroleptics. Concern about side effects of typical neuroleptics has increased popularity of atypical agents, though evidence for better efficacy or tolerability is not yet available. Neuroleptics may be particularly useful in patients who have concomitant psychosis or aggressive behavior. Depression in HD responds well to treatment with standard antidepressants. Valproate, carbamazepine, and selective serotonin reuptake inhibitors have been used for the management of mood instability, mania and irritability.

There is conflicting evidence on the utility of cholinesterase inhibitors for cognitive impairment in $\mathrm{HD}$, and no study has suggested a substantial benefit $[8,9]$.

\section{Treatment \\ Treatment of Chorea \\ Antidopaminergic agents}

Tetrabenazine (TBZ): In a double-blind, placebo controlled, 12-week study conducted by the Huntington Study Group, 84 patients with HD received TBZ up to $100 \mathrm{mg}$ daily or placebo. The mean improvement of baseline chorea in TBZ-treated patients was 23.5\% (5.0 units in Unified Huntington Disease Rating Scale (UHDRS)-derived chorea scores vs. 1.5 units in placebo arm)[10, Class I]. In an open-label extension of this study, patients received up to $200 \mathrm{mg}$ of TBZ daily for up to 80 weeks. The total maximal chorea score improved by 4.6 UHDRS units among 45 patients who completed 80 weeks of study intervention [11, Class IV]. Kenny et al. evaluated short-term effects of TBZ in HD patients in an open-label observational study [12, Class IV]. The mean improvement in UHDRS chorea score was $42.4 \% \pm 17.8 \%$ and persisted for an average of 5.4 hours. Several other studies confirmed the effectiveness of TBZ in the treatment of chorea [13,14].

Standard dosage: starting dose $25.5 \mathrm{mg}$ daily; maximum daily dose $100 \mathrm{mg}$, dosed up to 3 times per day.

Contraindications: Actively suicidal ideations, untreated or inadequately treated depression, hepatic function impairment, therapy with Monoamine Oxidase Inhibitors (MAOIs), reserpine therapy.

Main drug interactions: Concomitant treatment with fluoxetine and paroxetine requires reduction of daily TBZ dose by 50\%. Concomitant administration of tricyclic antidepressants may accentuate adverse effects of TBZ.

Main side effects: Depression, drowsiness, anxiety, parkinsonism, fatigue, akathisia, gastrointestinal distress, and rarely neuroleptic malignant syndrome.

Special points: The only U.S. Food and Drug Administration-approved agent for the symptomatic management of HD. A genotyping for CYP2D6 activity is recommended for patients taking TBZ daily doses $>50 \mathrm{mg}$.

Cost*: tab; strength: $12.5 \mathrm{mg}$; quantity $112 \mathrm{~s}$; average whole sale price $\$ 4,176.02$

\section{Antipsychotic agents}

Haloperidol: Koller et al. investigated 13 HD patients treated with haloperidol using daily doses of 2-80 $\mathrm{mg}$ [15, Class III]. Haloperidol treatment decreased chorea in all patients. Mean abnormal involuntary score decreased from $21.2 \pm 7.2$ pre-treatment to $8.5 \pm 3.0$ posttreatment. Girottiet al. investigated the effects of haloperidol on chorea in $18 \mathrm{HD}$ patients 
[16, Class III]. Haloperidol reduced abnormal involuntary movements as assessed by the abnormal involuntary movement score form $311.22 \pm 259.9$ pre-treatment to $131 \pm 141.26$ post-treatment.

Contraindications: Haloperidol is contraindicated in severe central nervous system depression or comatose states from any cause

Main drug interactions: A caution is advised when prescribing to a patient with QTprolongation conditions. Increased haloperidol concentrations have been reported when haloperidol was given concomitantly with drugs characterized as substrates or inhibitors of CYP3A4 or CYP2D6 isoenzymes, such as itraconazole, nefazodone, buspirone, venlafaxine, alprazolam, fluvoxamine, quinidine, fluoxetine, sertraline, chlorpromazine, and promethazine. Co-administration with rifampin or carbamazepine has been associated with a significant reduction of haloperidol plasma levels.

Main side effects: Tachycardia, hypotension, hypertension, extrapyramidal symptoms, withdrawal emergent neurological syndrome, tardive syndromes, insomnia, restlessness, anxiety, agitation, depression, confusion, lactation, gynecomastia, impotence, dry mouth, blurred vision, urinary retention.

Cost: tab; $1 \mathrm{mg} ; 100 \mathrm{~s} ; \$ 7.95$

* Costs estimates are based on the Red Book, PDR Network LLC, 2010 Edition, Montvale, NJ

Pimozide: Girottiet al. investigated effects of pimozide on chorea in 18 HD patients [16, Class III]. Haloperidol reduced abnormal involuntary movements as assessed by the abnormal involuntary movement scores from $393.36 \pm 255.51$ pre-treatment to $241.45 \pm 252.22$ post-treatment. Combination therapy with pimozide and tetrabenazine may be effective in improving chorea associated with HD, according to a small pilot study [17, Class IV].

Contraindications: In the treatment of simple tics or tics other than those associated with Tourette's Disorder; in patients with congenital long QT syndrome, history of cardiac arrhythmias, co-administration of other drugs which prolong the QT interval, severe central nervous system depression or comatose states from any cause; in patients receiving the macrolide antibiotics (e.g., clarithromycin, erythromycin, azithromycin, dirithromycin, and troleandomycin), escitalopram, paroxetine and other strong CYP 2D6 inhibitors, sertraline, itraconazole, ketokonazole, ritonavir, saquinovir, indinavir, and nelfinavir, nefazodone.

Main drug interactions: Additive effects on QT prolongation may be expected when coadministering pimozide with pheothiazines, tricyclic antidepressants or antiarrhythmic agents.

Main side effects: dry mouth, constipation, sedation, tardive dyskinesia, parkinsonism, depression, extrapyramidal symptoms, neuroleptic malignant syndrome.

Cost: tab; 1mg; 100s; $\$ 121.83$

Clozapine: Van Vught et al. investigated 33 HD patients in a double-blind, placebocontrolled trial of clozapine. A treatment with clozapine was associated with improved UHDRS chorea scores of -4.0 in the active treatment group vs. -0.3 in the placebo group $[18$, Class I]. Several other reports confirmed beneficial effects of clozapine on chorea [19, 
20, Class IV]. Colosimo et al., however, did not find clozapine effective for the treatment of chorea in a small retrospective study of 8 HD patients [21, Class IV].

Contraindications: Clozapine is contraindicated in patients with myeloproliferative disorders, uncontrolled epilepsy, paralytic ileus, or a history of clozapine-induced agranulocytosis or severe granulocytopenia.

Main drug interactions: Clozapine should be used with caution when coadministered with medications known to prolong the QTc interval. Concomitant administration of drugs known to induce cytochrome P450 enzymes may decrease the plasma levels of Clozapine. A reduced Clozapine dose should be considered when clozapine is combined with fluvoxamine and paroxetine.

Main side effects: sedation, dizziness, headache, tremor, salivation, sweating, dry mouth, visual disturbances, tachycardia, hypotension, syncope, constipation and nausea.

Special points: risk for agranulocytosis, seizures, myocarditis, syncope and increased risk of death in elderly patients with dementia-related psychosis.

Cost: tab; 25mg; 100s; 129.43

Olanzapine: In an open-label, prospective study of olanzapine for the treatment of motor symptoms of $\mathrm{HD}$, Bonelli et al. reported statistically significant reductions in chorea. UHDRS chorea scores improved from $13.4 \pm 6.8$ before treatment to $6.9 \pm 5.0$ after 14-day treatment with olanzapine [22, Class IV]. Several case reports confirmed the efficacy of olanzapine in the reduction of chorea [23-30, Class IV].

Contraindications: none specific

Main drug interactions: Drugs that induce CYP1A2 or glucuronyl transferase enzymes (e.g., carbamazepine, omeprazole, rifampin) may reduce olanzapine plasma levels. Drugs that inhibit CYP1A2 (e.g., estrogens, fluvoxamine) may elevate olanzapine plasma levels.

Main side effects: sedation, extrapyramidal symptoms, akathisia, asthenia, dizziness, fatigue, insomnia, increased appetite, dry mouth, nausea, elevated liver enzymes, weight gain.

Cost: tab; 2.5mg; 100s; $\$ 877.20$

Ziprasidone: In a case series of 3 HD patients, Bonelli et al. reported significant improvements of chorea with ziprasidone daily doses 80-160mg [31, Class IV].

Contraindications: Ziprasidone is contraindicated in patients with a known history of QT prolongation, recent acute myocardial infarction, and uncompensated heart failure.

Main drug interactions: Co-administration of carbamazepine may lead to a decrease in plasma levels of ziprasidone. Co-administration of ketoconazole may lead to increased plasma concentrations of ziprasidone.

Main side effects: Somnolence, extrapyramidal symptoms, dizziness, akathisia, asthenia, vomiting, weight gain, anxiety.

Cost: cap; 20mg; 60s; $\$ 479.00$

Aripiprazole: Brusa et al. investigated the effects of aripiprazole on chorea in 6 patients with HD [32, Class IV]. Treatment with aripiprazole resulted in a reduction of UHDRS 
chorea score of 5.4 units. In a case series of 3 HD patients, Ciammola et al. reported significant improvements in chorea with daily aripiprazole dose of 7.5-15 mg [33, Class IV].

Contraindications: none specific.

Main drug interactions: Drugs that induce CYP3A4 (e.g., carbamazepine) could cause an increase in aripiprazole clearance and lower blood levels. Inhibitors of CYP3A4 (e.g., ketoconazole) or CYP2D6 (e.g., quinidine, fluoxetine, or paroxetine) can inhibit aripiprazole elimination and cause increased blood levels. When ketoconazole and quinidine are given concomitantly with aripiprazole, the aripiprazole dose should be reduced to one-half of its normal dose. When carbamazepine is added to aripiprazole therapy, aripiprazole dose should be doubled.

Main side effects: nausea, vomiting, constipation, headache, dizziness, akathisia, anxiety, insomnia, restlessness, tremor, extrapyramidal disorder.

Special points: risk for increased mortality in elderly patients with dementia-related psychosis, clinical worsening of depression and suicide, neuroleptic malignant syndrome, tardive dyskinesia, hyperglycemia, orthostatic hypotension, leucopenia/neutropenia/ agranulocytosis, seizures, body temperature regulation.

Cost: tab; 2mg; 30s; $\$ 514.04$

Risperidone: Parsa et al. reported marked improvement in choreoathetoid involuntary movements after introducing and titrating risperidone to a daily dose of $6 \mathrm{mg}$ in a HD patient already treated with clozapine for psychiatric manifestation of the disease [34, Class IV]. Dallacchio et al. reported similar beneficial effects of risperidone on chorea in 4 HD patients treated with daily doses of $6 \mathrm{mg}$ [35, Class IV].

Contraindications: none specific

Main drug interactions: Coadministration of carbamazepine and similar enzyme inducers (e.g., phenytoin, rifampin, and phenobarbital) may result in decreased plasma concentrations of risperidone. Fluoxetine and paroxetine have been shown to increase the plasma concentration of risperidone. Cimetidine and ranitidine may increase the bioavailability of risperidone.

Main side effects: somnolence, increased appetite, fatigue, insomnia, sedation, parkinsonism, akathisia, cough, drooling, rhinorrhea, dry mouth, dizziness, nausea, anxiety, headache, nasal congestion, tremor.

Cost: tab; 0.5mg; 60s; $\$ 85.64$

Quetiapine: Treatment with quetiapine was associated with reduction in choreiform movements in a case report of a HD patient [36, Class IV].

Contraindications: none specific

Main drug interactions: The use of quetiapine should be avoided in combination with drugs known to increase QT interval. Increased doses of quetiapine may be required in patients receiving quetiapine and phenytoin, or other hepatic enzyme inducers (e.g., carbamazepine, barbiturates, rifampin, glucocorticoids). Reduced dosage is indicated when quetiapine is administered with inhibitors of cytochrome P450 3A. 
Main side effects: somnolence, dizziness, dry mouth, constipation, weight gain, dyspepsia, hypotension.

Cost: tab; 25mg; 100s; $\$ 328.32$

\section{N-Methyl-D-Aspartic Acid Receptor Antagonists}

Amantadine: Investigations of amantadine for the treatment of chorea have revealed conflicting results. In a 4-week study of $24 \mathrm{HD}$ patients, treatment with oral amantadine in doses up to $400 \mathrm{mg} /$ day resulted in significant suppression of chorea [37, Class I]. Intravenous administration of amantadine in 9 patients was associated with significant improvements in dyskinesia scores [38, , Class II]. Several double-blind placebo-controlled trials, failed to demonstrate beneficial effects of amantadine on chorea of HD [39, 40, Class II/III].

Contraindications: none specific

Main drug interactions: Co-administration of quinine or quinidine with amantadine reduces the renal clearance of amantadine by about $30 \%$.

Main side effects: nausea, dizziness (lightheadedness), insomnia, anxiety, hallucinations, confusion, anorexia, dry mouth, constipation, ataxia, livedo reticularis, peripheral edema, orthostatic hypotension, headache, somnolence, nervousness, dream abnormality, agitation, dry nose, diarrhea and fatigue.

Cost: tab; 100mg; 100s; $\$ 120.03$

Memantine: In a small open-label study of $9 \mathrm{HD}$ patients, treatment with memantine at $20 \mathrm{mg} /$ day resulted in significant improvements in chorea scores form baseline $11.5 \pm 6.3$ to $4.8 \pm 3.8$ at three months follow up [41, Class IV].

Contraindications: none specific.

Main drug interactions: none significant.

Main side effects: dizziness, headache, constipation, confusion, cough, hypertension.

Cost: tab; 5mg; 60s; $\$ 199.08$

\section{Omega-3 Fatty Acids}

Ethyl-eicosapentaenoic acid (EPA): A double-blind, placebo-controlled study in patients with early stage HD tested the effects of ethyl-EPA (2g/day) vs. placebo on Total Motor Score-4 (subset of UHDRS excluding 8 items from the TMS) [42, Class I]. After 12 months of treatment, no significant differences in TMS-4 were found between ethyl-EPA and placebo-treated groups. In the subset of HD patients with 44 or fewer CAG repeats significant improvements in TMS-4 scores were observed. Subsequently, a large phase III study of ethyl-EPA for treatment of chorea, enrolled 316 HD patients in North America [43, Class I]. A similar study of $290 \mathrm{HD}$ patients was conducted in Europe. Both studies failed to demonstrated beneficial effects of ethyl-EPA on TMS-4 (primary endpoint) or total chorea score, TMS, CGI severity and global improvement score (secondary endpoints).

Contraindications: none specific.

Main drug interactions: EPA may slow blood coagulation. 
Main side effects: diarrhea, heartburn, itching, nosebleed, and joint, back, and muscle pain.

\section{Treatment of Cognitive Dysfunction Associated with HD}

Rivastigmine-Treatment with rivastigmine ( 3 to $6 \mathrm{mg}$ daily) was associated with improvements of cognitive function, as measured by MMSE, in 11 patients with HD followed for 24 months [44, Class III].

Contraindications: none specific.

Main drug interactions: none significant

Main side effects: nausea, vomiting, anorexia, dizziness, headache, fatigue, insomnia, confusion.

Cost: cap; 3mg; 100s; $\$ 441.04$

Donepezil-A 12-week, randomized, double-blind placebo controlled trial of donepezil failed to demonstrate beneficial effects of donepezil on cognitive performance in HD [45, Class I]. Similar findings were reported in an open-label study of donepezil for cognitive dysfunction associated with HD [46, Class IV].

Contraindications: none specific.

Main drug interactions: Inducers of CYP 2D6 and CYP 3A4 (e.g., phenytoin, carbamazepine, dexamethasone, rifampin, and phenobarbital) could increase the rate of elimination of donepezil.

Main side effects: diarrhea, nausea, vomiting, dizziness, headache, agitation, anorexia.

Cost: tab; 5mg; 100s; $\$ 802.78$

\section{Treatment of Behavioral Disturbances Associated with HD \\ Depression}

Fluoxetine: In a randomized, double-blind, placebo-controlled clinical trial of 30 participants with early HD and Hamilton Depression Inventory $>16$, treatment with fluoxetine $(20 \mathrm{mg} /$ day) was associated with a slight reduction in agitation [47, Class I].

Contraindications: The use of fluoxetine is contraindicated with MAOIs, pimozide, and thioridazine.

Main drug interactions: Caution is advised when fluoxetine is co-administered with other drugs that may affect the serotonergic neurotransmitter systems. Fluoxetine inhibits the activity of CYP2D6, and may make individuals with normal CYP2D6 metabolic activity resemble a poor metabolizer. The dose of TCAs may need to be reduced if when coadministered with fluoxetine. Lithium levels should be monitored when co-administered with fluoxetine.

Main side effects: nausea, headache, insomnia, anxiety, somnolence, dizziness, asthenia, tremor, dry mouth.

Cost: cap; 20mg; 100s; $\$ 248.40-556.50$ 
Venlafaxine: In an open label study including $26 \mathrm{HD}$ participants with co-existent major depression, treatment with Venlafaxine ER ( $75 \mathrm{mg} /$ day) was associated with significant improvement in depression assessed by the Beck Depression Inventory and the Hamilton Rating Scale [48, Class IV]. Approximately one in five patients, however, developed significant venlafaxine-related side effects, mainly nausea and irritability.

Contraindications: In patients taking MAOIs or in patients who have taken MAOIs within the preceding 14 days due to the risk of serious drug interactions with SNRI or SSRI treatment or with other serotonergic drugs.

Main drug interactions: Concomitant use of CYP3A4 inhibitors may increase levels of venlafaxine. Caution is advised when venlafaxine is co-administered with drugs that may affect the serotonergic neurotransmitter systems, such as triptans, SSRIs, other SNRIs, linezolid, lithium, tramadol, or St. John's Wort.

Main side effects: asthenia, sweating, nausea, constipation, anorexia, vomiting, somnolence, dry mouth, dizziness, nervousness, anxiety, tremor, and blurred vision as well as abnormal ejaculation/orgasm and impotence in men.

Cost: tab; 37.5mg; 30s; $\$ 113.83$

Mirtazapine: Mirtazapine has been reported efficacious for treating severe depression and suicidal ideations in a 32-year old woman with HD at the doses of 60-90mg/day [49, Class IV].

Contraindications: The concomitant use of mirtazapine orally disintegrating tablets and a MAOI is contraindicated. Mirtazapine should not be used within 14 days of initiating or discontinuing therapy with a MAOI.

Main drug interactions: When phenytoin, carbamazepine, or another inducer of hepatic metabolism is added to mirtazapine therapy, the mirtazapine dose may have to be increased. The mirtazapine dose may have to be decreased when concomitant treatment with cimetidine or ketoconazole is started.

Main side effects: somnolence, increased appetite, weight gain, dizziness, dry mouth, constipation.

Cost: tab; 30mg; 30s; $\$ 37.95$

Clozapine: Clozapine has been reported to be efficacious for treating depression in a 32year old woman with HD at the daily dose of $175 \mathrm{mg}$ [50, Class IV].

Contraindications: Clozapine is contraindicated in patients with myeloproliferative disorders, uncontrolled epilepsy, paralytic ileus, or a history of clozapine-induced agranulocytosis or severe granulocytopenia.

Main drug interactions: Clozapine should be used with caution when coadministered with medications known to prolong the QT interval. Concomitant administration of drugs known to induce cytochrome P450 enzymes may decrease the plasma levels of clozapine. A reduced clozapine dose should be considered when clozapine is combined with fluvoxamine and paroxetine. 
Main side effects: drowsiness/sedation, dizziness/vertigo, headache, tremor, salivation, sweating, dry mouth, visual disturbances, tachycardia, hypotension, syncope, constipation and nausea.

Special points: risk for agranulocytosis, seizures, myocarditis, syncope and increased risk of death in elderly patients with dementia-related psychosis.

Cost: tab; 25mg; 100s; $\$ 129.43$

\section{Psychosis}

Risperidone: Two case reports documented beneficial effects of risperidone in treatment of psychotic symptoms associated with HD [51, 52, Class IV].

Contraindications: none specific

Main drug interactions: Coadministration of carbamazepine and similar enzyme inducers (e.g., phenytoin, rifampin, and phenobarbital) may result in decreased plasma concentrations of risperidone. Fluoxetine and paroxetine have been shown to increase the plasma concentration of risperidone. Cimetidine and ranitidine may increase the bioavailability of risperidone.

Main side effects: somnolence, increased appetite, fatigue, insomnia, sedation, parkinsonism, akathisia, cough, drooling, rhinorrhea, dry mouth, dizziness, nausea, anxiety, headache, nasal congestion, tremor.

Cost: tab; 1mg; 60s; \$91.04

\section{Irritability, Agitation}

Olanzapine: In an open-label, six-month study, olanzapine (5mg/day) was associated with a significant improvement in the behavioral symptoms in 11 participants with HD. In this study, behavioral symptoms were assessed by the UHDRS behavioral score of items regarding depression, anxiety and irritability [53, Class IV]. Similarly, Paleacu et al. reported significant improvements in the UHDRS derived behavioral scores in $11 \mathrm{HD}$ patients treated with olanzapine (median dose $10 \mathrm{mg} /$ day) over the period of $9.8 \pm 5.9$ months [54, Class IV].

Contraindications: none specific

Main drug interactions: Drugs that induce CYP1A2 or glucuronyl transferase enzymes (e.g., carbamazepine, omeprazole, rifampin) may reduce olanzapine plasma levels. Drugs that inhibit CYP1A2 (e.g., estrogens, fluvoxamine) may elevate olanzapine plasma levels.

Main side effects: sedation, extrapyramidal symptoms, akathisia, asthenia, dizziness, fatigue, insomnia, increased appetite, dry mouth, nausea, elevated liver enzymes, weight gain.

Cost: tab; 2.5mg; 100s; $\$ 877.20$

Quetiapine: Irritability, agitation and insomnia improved significantly after adding quetiapine at doses of 150-300 mg per day to the medication regimens of five HD patients with co-existent behavioral dysfunction [55, Class IV].

Contraindications: none specific 
Main drug interactions: The use of quetiapine should be avoided in combination with drugs known to increase QT interval. Increased doses of quetiapine may be required in patients receiving quetiapine and phenytoin, or other hepatic enzyme inducers (e.g., carbamazepine, barbiturates, rifampin, glucocorticoids). Reduced dosage is indicated when quetiapine is administered with inhibitors of cytochrome P450 3A.

Main side effects: somnolence, dizziness, dry mouth, constipation, weight gain, dyspepsia, hypotension.

Cost: tab; 25mg; 100s; $\$ 328.32$

Sertraline: Beneficial effects of sertraline $(100 \mathrm{mg} /$ day $)$ on irritability and aggressiveness in two HD patients were reported by Ranen and colleagues [56, Class IV].

Contraindications: Concomitant use in patients taking MAOIs or pimozide is contraindicated.

Main drug interactions: Caution is advised during co-administration with warfarin due to observed increase in prothrombin time after initiating sertraline. Concomitant use of a drug metabolized by P450 2D6 with sertraline may require lower doses than usually prescribed for the other drug. Caution is advised when sertraline is coadministered with other drugs that may affect the serotonergic neurotransmitter systems, such as triptans, linezolid, lithium, tramadol, or St. John's Wort.

Main side effects: somnolence, dry mouth, dizziness, tremor, fatigues, diarrhea, nausea, dyspepsia, insomnia.

Cost: tab; 100mg; 30s; $\$ 85.26$

Buspirone: Three case reports documented the successful management of irritability and aggressive behavior with buspirone at the dose range $20-60 \mathrm{mg} /$ day [57-59, Class IV].

Contraindications: none specific

Main drug interactions: It is recommended that buspirone not be used concomitantly with MAOIs. Drugs that inhibit CYP3A4 (e.g. such as ketoconazole, ritonavir) may inhibit buspirone metabolism and increase plasma concentrations of buspirone while substances that induce CYP3A4, (e.g. dexamethasone, phenytoin, phenobarbital, carbamazepine), may increase the rate of Buspirone metabolism.

Main side effects: dizziness, nausea, headache, nervousness, lightheadedness.

Cost: tab; 5mg; 90s; $\$ 72.32$

Valproate: The combination of valproate (1500 $\mathrm{mg} /$ day), given as a mood stabilizer, with olanzapine has been reported helpful in relieving agitation and aggression associated with HD [28, Class IV]. This combination allows for a reduced dose of an antipsychotic drug and therefore lessens chances for adverse drug effects.

Contraindications: Valproate is contraindicated in patients with known urea cycle disorders and should not be administered to patients with hepatic disease or significant hepatic dysfunction. 
Main drug interactions: Drugs that affect the level of expression of hepatic enzymes, particularly glucuronosyltransferases, may increase the clearance of valproate (e.g. phenytoin, carbamazepine, and phenobarbital). A significant reduction in serum valproic acid concentration has been reported in patients receiving carbapenem antibiotics (e.g., ertapenem, imipenem, meropenem).

Main side effects: Life threatening adverse reactions reported in patients receiving valproate include hepatotoxicity, teratogenicity and pancreatitis (boxed warnings by FDA). Most common side effects include: asthenia, nausea, vomiting, thrombocytopenia, tremor, somnolence, dizziness, insomnia, alopecia, blurred vision, peripheral edema.

Cost: tab; 250mg; 100s; $\$ 52.50$

Propranolol: Propranolol has been reported as effective in controlling aggressive behavior in three HD patients at daily doses of 30-240mg [60, Class IV]. In another case report, propranolol was, however, associated with paradoxical worsening of HD-associated aggression [61, Class IV].

Contraindications: cardiogenic shock, sinus bradycardia, greater than first-degree block, bronchial asthma.

Main drug interactions: Blood levels and/or toxicity of propranolol may be increased by coadministration with substrates or inhibitors of CYP2D6, CYP1A2, CYP2C19. Blood levels of propranolol may be decreased by co-administration with inducers such as rifampin, ethanol, phenytoin, and phenobarbital. Propranolol can inhibit the metabolism of diazepam, resulting in increased concentrations of diazepam and its metabolites.

Main side effects: nausea, irritability, insomnia, fatigue, vivid dreams.

Cost: tab; 60mg; 60s; $\$ 93.72$

\section{Other Interventions}

Deep Brain Stimulation (DBS): Several publications reported effects of DBS on motor and neurocognitive performance in HD. A long-term follow up of two HD patients who underwent bilateral globus pallidus interna DBS revealed sustained improvement in chorea, but continuing decline in gait, bradykinesia, and cognitive performance [62, Class IV]. Improvements in chorea with concomitant decline in ambulation and cognition have been reported is several additional case reports of HD patients post DBS surgery [63-65, Class IV].

\section{References}

1. The Huntington's Disease Collaborative Research Group. A novel gene containing a trinucleotide repeat that is expanded and unstable on Huntington's disease chromosomes. Cell. 1993; 72(6):971983. [PubMed: 8458085]

2. Mahant N, McCusker EA, Byth K, Graham S. Huntington's disease: clinical correlates of disability and progression. Neurology. 2003; 61(8):1085-1092. [PubMed: 14581669]

3. Di Maio L, Squitieri F, Napolitano G, et al. Onset symptoms in 510 patients with Huntington's disease. J Med Genet. 1993; 30(4):289-292. [PubMed: 8487272]

4. Paulsen JS, Ready RE, Hamilton JM, et al. Neuropsychiatric aspects of Huntington's disease. J Neurol Neurosurg Psychiatry. 2001; 71(3):310-314. [PubMed: 11511702]

5. Litvan I, Paulsen JS, Mega MS, Cummings JL. Neuropsychiatric assessment of patients with hyperkinetic and hypokinetic movement disorders. Arch Neurol. 1998; 55(10):1313-1319.

[PubMed: 9779658] 
6. Ho AK, Sahakian BJ, Brown RG, et al. Profile of cognitive progression in early Huntington's disease. Neurology. 2003; 61(12):1702-1706. [PubMed: 14694033]

7. Bamford KA, Caine ED, Kido DK, et al. A prospective evaluation of cognitive decline in early Huntington's disease: functional and radiographic correlates. Neurology. 1995; 45(10):1867-1873. [PubMed: 7477984]

8. Fernandez HH, Friedman JH, Grace J, Beason-Hazen S. Donepezil for Huntington's disease. Mov Disord. 2000; 15(1):173-176. [PubMed: 10634264]

9. de Tommaso M, Specchio N, Sciruicchio V, et al. Effects of rivastigmine on motor and cognitive impairment in Huntington's disease. Mov Disord. 2004; 19(12):1516-1518. [PubMed: 15390067]

10. Huntington Study Group. Tetrabenazine as antichorea therapy in Huntington disease: a randomized controlled trial. Neurology. 2006; 66(3):366-372. [PubMed: 16476934]

11. Frank S. Tetrabenazine as anti-chorea therapy in Huntington disease: an open-label continuation study. Huntington Study Group/TETRA-HD Investigators. BMC Neurol. 2009; 9:62. [PubMed: 20021666]

12. Kenney C, Hunter C, Davidson A, Jankovic J. Short-term effects of tetrabenazine on chorea associated with Huntington's disease. Mov Disord. 2007; 22(1):10-13. [PubMed: 17078062]

13. Jankovic J, Beach J. Long-term effects of tetrabenazine in hyperkinetic movement disorders. Neurology. 1997; 48(2):358-362. [PubMed: 9040721]

14. Ondo WG, Tintner R, Thomas M, Jankovic J. Tetrabenazine treatment for Huntington's diseaseassociated chorea. Clin Neuropharmacol. 2002; 25(6):300-302. [PubMed: 12469001]

15. Koller WC, Trimble J. The gait abnormality of Huntington's disease. Neurology. 1985; 35(10): 1450-1454. [PubMed: 3162109]

16. Girotti F, Carella F, Scigliano G, et al. Effect of neuroleptic treatment on involuntary movements and motor performances in Huntington's disease. J Neurol Neurosurg Psychiatry. 1984; 47(8):848852. [PubMed: 6147393]

17. McArthur AW, Pollock M, Smidt NA. Combined therapy with tetrabenazine and pimozide in Huntington's chorea: pilot study. N Z Med J. 1976; 83(558):114-116. [PubMed: 131917]

18. van Vugt JP, Siesling S, Vergeer M, et al. Clozapine versus placebo in Huntington's disease: a double blind randomised comparative study. J Neurol Neurosurg Psychiatry. 1997; 63(1):35-39. [PubMed: 9221965]

19. Bonuccelli U, Ceravolo R, Maremmani C, et al. Clozapine in Huntington's chorea. Neurology. 1994; 44(5):821-823. [PubMed: 8190281]

20. Vallette N, Gosselin O, Kahn JP. [Efficacy of clozapine in the course of Huntington chorea: apropos of a clinical case]. Encephale. 2001; 27(2):169-171. [PubMed: 11407269]

21. Colosimo C, Cassetta E, Bentivoglio AR, Albanese A. Clozapine in Huntington's disease. Neurology. 1995; 45(5):1023-1024. [PubMed: 7746380]

22. Bonelli RM, Mahnert FA, Niederwieser G. Olanzapine for Huntington's disease: an open label study. Clin Neuropharmacol. 2002; 25(5):263-265. [PubMed: 12410058]

23. Bogelman G, Hirschmann S, Modai I. Olanzapine and Huntington's disease. J Clin Psychopharmacol. 2001; 21(2):245-246. [PubMed: 11270928]

24. Bonelli RM, Niederwieser G, Diez J, Koltringer P. Riluzole and olanzapine in Huntington's disease. Eur J Neurol. 2002; 9(2):183-184. [PubMed: 11882065]

25. Bonelli RM, Niederwieser G, Tribl GG, Koltringer P. High-dose olanzapine in Huntington's disease. Int Clin Psychopharmacol. 2002; 17(2):91-93. [PubMed: 11890191]

26. Dipple HC. The use of olanzapine for movement disorder in Huntington's disease: a first case report. J Neurol Neurosurg Psychiatry. 1999; 67(1):123-124. [PubMed: 10454874]

27. Etchebehere EC, Lima MC, Passos W, et al. Brain SPECT imaging in Huntington's disease before and after therapy with olanzapine. Case report. Arq Neuropsiquiatr. 1999; 57(3B):863-866. [PubMed: 10751925]

28. Grove VE Jr. Quintanilla J, DeVaney GT. Improvement of Huntington's disease with olanzapine and valproate. N Engl J Med. 2000; 343(13):973-974. [PubMed: 11012330]

29. Jimenez-Jimenez FJ, de Toledo M, Puertas I, et al. [Olanzapine improves chorea in patients with Huntington's disease]. Rev Neurol. 2002; 35(6):524-525. [PubMed: 12389168] 
30. Laks J, Rocha M, Capitao C, et al. Functional and motor response to low dose olanzapine in Huntington's disease: case report. Arq Neuropsiquiatr. 2004; 62(4):1092-1094. [PubMed: 15608976]

31. Bonelli RM, Mayr BM, Niederwieser G, et al. Ziprasidone in Huntington's disease: the first case reports. J Psychopharmacol. 2003; 17(4):459-460. [PubMed: 14870962]

32. Brusa L, Orlacchio A, Moschella V, et al. Treatment of the symptoms of Huntington's disease: preliminary results comparing aripiprazole and tetrabenazine. Mov Disord. 2009; 24(1):126-129. [PubMed: 19170197]

33. Ciammola A, Sassone J, Colciago C, et al. Aripiprazole in the treatment of Huntington's disease: a case series. Neuropsychiatr Dis Treat. 2009; 5:1-4. [PubMed: 19557093]

34. Parsa MA, Szigethy E, Voci JM, Meltzer HY. Risperidone in treatment of choreoathetosis of Huntington's disease. J Clin Psychopharmacol. 1997; 17(2):134-135. [PubMed: 10950488]

35. Dallocchio C, Buffa C, Tinelli C, Mazzarello P. Effectiveness of risperidone in Huntington chorea patients. J Clin Psychopharmacol. 1999; 19(1):101-103. [PubMed: 9934953]

36. Bonelli RM, Niederwieser G. Quetiapine in Huntington's disease: a first case report. J Neurol. 2002; 249(8):1114-1115. [PubMed: 12420714]

37. Verhagen Metman L, Morris MJ, Farmer C, et al. Huntington's disease: a randomized, controlled trial using the NMDA-antagonist amantadine. Neurology. 2002; 59(5):694-699. [PubMed: 12221159]

38. Lucetti C, Del Dotto P, Gambaccini G, et al. IV amantadine improves chorea in Huntington's disease: an acute randomized, controlled study. Neurology. 2003; 60(12):1995-1997. [PubMed: 12821751]

39. Heckmann JM, Legg P, Sklar D, et al. IV amantadine improves chorea in Huntington's disease: an acute randomized, controlled study. Neurology. 2004; 63(3):597-598. author reply 597-598. [PubMed: 15304616]

40. O'Suilleabhain P, Dewey RB Jr. A randomized trial of amantadine in Huntington disease. Arch Neurol. 2003; 60(7):996-998. [PubMed: 12873857]

41. Ondo WG, Mejia NI, Hunter CB. A pilot study of the clinical efficacy and safety of memantine for Huntington's disease. Parkinsonism Relat Disord. 2007; 13(7):453-454. [PubMed: 17046312]

42. Puri BK, Leavitt BR, Hayden MR, et al. Ethyl-EPA in Huntington disease: a double-blind, randomized, placebo-controlled trial. Neurology. 2005; 65(2):286-292. [PubMed: 16043801]

43. Randomized controlled trial of ethyl-eicosapentaenoic acid in Huntington disease: the TREND-HD study. Arch Neurol. 2008; 65(12):1582-1589. [PubMed: 19064745]

44. de Tommaso M, Difruscolo O, Sciruicchio V, et al. Two years' follow-up of rivastigmine treatment in Huntington disease. Clin Neuropharmacol. 2007; 30(1):43-46. [PubMed: 17272969]

45. Cubo E, Shannon KM, Tracy D, et al. Effect of donepezil on motor and cognitive function in Huntington disease. Neurology. 2006; 67(7):1268-1271. [PubMed: 17030764]

46. Fernandez HH, Friedman JH, Grace J, Beason-Hazen S. Donepezil for Huntington's disease. Mov Disord. 2000; 15(1):173-176. [PubMed: 10634264]

47. Como PG, Rubin AJ, O'Brien CF, et al. A controlled trial of fluoxetine in nondepressed patients with Huntington's disease. Mov Disord. 1997; 12(3):397-401. [PubMed: 9159735]

48. Holl AK, Wilkinson L, Painold A, et al. Combating depression in Huntington's disease: effective antidepressive treatment with venlafaxine XR. Int Clin Psychopharmacol. 2010; 25(1):46-50. [PubMed: 19996754]

49. Bonelli RM. Mirtazapine in suicidal Huntington's disease. Ann Pharmacother. 2003; 37(3):452. [PubMed: 12639181]

50. Sajatovic M, Verbanac P, Ramirez LF, Meltzer HY. Clozapine treatment of psychiatric symptoms resistant to neuroleptic treatment in patients with Huntington's chorea. Neurology. 1991; 41(1): 156. [PubMed: 1670739]

51. Erdemoglu AK, Boratav C. Risperidone in chorea and psychosis of Huntington's disease. Eur J Neurol. 2002; 9(2):182-183. [PubMed: 11882064]

52. Madhusoodanan S, Brenner R. Use of risperidone in psychosis associated with Huntington's disease. Am J Geriatr Psychiatry. 1998; 6(4):347-349. [PubMed: 9793585] 
53. Squitieri F, Cannella M, Piorcellini A, et al. Short-term effects of olanzapine in Huntington disease. Neuropsychiatry Neuropsychol Behav Neurol. 2001; 14(1):69-72. [PubMed: 11234911]

54. Paleacu D, Anca M, Giladi N. Olanzapine in Huntington's disease. Acta Neurol Scand. 2002; 105(6):441-444. [PubMed: 12027832]

55. Alpay M, Koroshetz WJ. Quetiapine in the treatment of behavioral disturbances in patients with Huntington's disease. Psychosomatics. 2006; 47(1):70-72. [PubMed: 16384811]

56. Ranen NG, Lipsey JR, Treisman G, Ross CA. Sertraline in the treatment of severe aggressiveness in Huntington's disease. J Neuropsychiatry Clin Neurosci. 1996; 8(3):338-340. [PubMed: 8854307]

57. Bhandary AN, Masand PS. Buspirone in the management of disruptive behaviors due to Huntington's disease and other neurological disorders. Psychosomatics. 1997; 38(4):389-391. [PubMed: 9217410]

58. Byrne A, Martin W, Hnatko G. Beneficial effects of buspirone therapy in Huntington's disease. Am J Psychiatry. 1994; 151(7):1097. [PubMed: 8010374]

59. Findling RL. Treatment of aggression in juvenile-onset Huntington's disease with buspirone. Psychosomatics. 1993; 34(5):460-461. [PubMed: 8140199]

60. Stewart JT, Mounts ML, Clark RL Jr. Aggressive behavior in Huntington's disease: treatment with propranolol. J Clin Psychiatry. 1987; 48(3):106-108. [PubMed: 2880838]

61. Stewart JT. Paradoxical aggressive effect of propranolol in a patient with Huntington's disease. J Clin Psychiatry. 1987; 48(9):385-386. [PubMed: 2957363]

62. Kang GA, Heath S, Rothlind J, Starr PA. Long-term follow-up of pallidal deep brain stimulation in two cases of Huntington's disease. J Neurol Neurosurg Psychiatry. 2011; 82(3):272-277. [PubMed: 20974647]

63. Biolsi B, Cif L, Fertit HE, et al. Long-term follow-up of Huntington disease treated by bilateral deep brain stimulation of the internal globus pallidus. J Neurosurg. 2008; 109(1):130-132. [PubMed: 18590443]

64. Fasano A, Mazzone P, Piano C, et al. GPi-DBS in Huntington's disease: results on motor function and cognition in a 72-year-old case. Mov Disord. 2008; 23(9):1289-1292. [PubMed: 18512756]

65. Moro E, Lang AE, Strafella AP, et al. Bilateral globus pallidus stimulation for Huntington's disease. Ann Neurol. 2004; 56(2):290-294. [PubMed: 15293283] 


\section{Opinion Statement}

Many pharmacological agents have been utilized in the treatment of Huntington disease (HD). Several excellent reviews about the treatment of HD are available. Formal treatment guidelines are however lacking. This is mainly the result of limited evidence available in the literature. Further, available treatment studies are frequently hard to compare due to variable outcomes/instruments used, differences in the study population, and confounding effects of complex medication regimens. Generally speaking, the treatment paradigm for an HD patient will depend on the constellation of three main clinical domains affected in HD: motor, behavioral/psychiatric, and cognitive. Symptoms within each of these domains remain dynamic throughout the course of HD. It is therefore necessary to monitor patients clinically and adjust drugs accordingly as the disease progresses.

The most commonly used chorea drugs are antipsychotics and tetrabenazine (TBZ). Antipsychotic drugs are preferred in patients with coexistent psychiatric/behavioral comorbidities as well as in the presence of depression. Amantadine may be considered in the treatment of chorea, but data supporting its effectiveness remain conflicting. Selective serotonin reuptake inhibitors (SSRIs) are the treatment of choice for irritability and obsessive-compulsive behaviors associated with HD. Antipsychotic agents and antiepileptic mood stabilizers may be used as add-on therapies. There is very limited evidence for the treatment of cognitive impairment associated with HD.

Each drug used in treatment of HD has a potential for causing significant side effects. It is, therefore, critical to assess the risk-benefit ratio on an individual basis, and carefully monitor patients throughout the course of treatment.

Non-pharmacological and surgical treatment strategies for HD have not been systematically explored. Despite the lack of evidence, behavioral interventions, as well as physical, occupational and speech therapies may provide additional benefits to a wide spectrum of disabilities associated with HD. 